\title{
Management plans for populations with normal-to-hypertensive blood pressures: risks and benefits of antihypertensive drug treatment in populations previously defined as having high-normal blood pressure
}

Sungha Park

Division of Cardiology, Severance Cardiovascular Hospital, Yonsei University College of Medicine, Seoul, Korea
Received: October 22, 2018 Accepted: December 14, 2018

\section{Correspondence to}

Sungha Park, M.D.

Division of Cardiology, Severance Cardiovascular Hospital, Yonsei University College of Medicine, 50-1 Yonsei-ro, Seodaemun-gu, Seoul 03722, Korea

Tel: +82-2-2228-1975

Fax: +82-2-393-6884

E-mail: shparko530@yuhs.ac

This paper was contributed by The Korean Society of Cardiology.
Recent changes in American and European guidelines on the management of arterial hypertension have caused a considerable shift in the landscape of hypertension management. The 2017 American College of Cardiology/American Heart Association/American Society of Hypertension guideline recommends an office visit blood pressure $(\mathrm{BP})>130 / 80 \mathrm{mmHg}$ as the new threshold for diagnosis of hypertension, and states that the treatment goal for all hypertensive patients should be lowered to $<130 / 80 \mathrm{mmHg}$. In contrast, the 2018 European guideline maintains the diagnostic threshold of hypertension at $140 / 90 \mathrm{mmHg}$. However, despite their differences in thresholds for diagnosis of hypertension, both guidelines are in agreement that treatment should be considered in patients with BPs in the range of 130 to $139 / 80$ to $89 \mathrm{mmHg}$ if they have high cardiovascular risk. The results from the Systolic Blood Pressure Intervention Trial (SPRINT) study and recent meta-analyses suggest that BP lowering with antihypertensive treatment may be beneficial in reducing cardiovascular event rates in subjects with high-normal BP or stage 1 hypertension according to the new American guideline. However, intensive BP lowering is associated with increased incidence of treatment-associated adverse events, and evidence suggests that BP lowering below $120 / 70 \mathrm{mmHg}$ increases the risk of cardiovascular events. In this review, we discuss the evidence supporting antihypertensive treatment in subjects with high-normal BP and discuss the specific subgroup of subjects that might benefit from BP lowering.

Keywords: Blood pressure; Hypertension; Treatment target; Cardiovascular diseases

\section{INTRODUCTION}

Recent changes in American and European guidelines on the management of arterial hypertension have caused a considerable shift in the landscape of hypertension management [1,2]. The 2017 American College of Cardiology/American Heart Association/American Society of Hypertension guideline recommends a new 
threshold for diagnosis of hypertension, i.e., an office visit blood pressure $(\mathrm{BP})>130 / 80 \mathrm{mmHg}$, and states that the treatment goal for all hypertensive patients should be lowered to < 130/80 $\mathrm{mmHg}$ [1]. In addition, the new American guideline recommends that drug treatment should be considered in high-risk patients with stage 1 hypertension, and in those with BPs in the range of 130/80 to $140 / 90 \mathrm{mmHg}$ and an arteriosclerotic vascular disease risk score of $>10 \%$ or cardiovascular disease (CVD) [1]. In contrast, the new 2018 European guideline maintains the diagnostic threshold of hypertension at $140 / 90 \mathrm{mmHg}[2]$. However, the European guideline recommends a strict target systolic blood pressure (SBP) goal of $\leq 130 \mathrm{mmHg}$ for most hypertensive patients, with the exception of those with chronic kidney disease (CKD) or advanced age ( $>65$ years); furthermore, the diastolic blood pressure (DBP) should be controlled in the range of 70 to $80 \mathrm{mmHg}$ [2]. In addition, the new European guideline suggests that drug treatment should be considered in patients with BPs in the range of 130 to $139 / 85$ to $89 \mathrm{mmHg}$ who are at high cardiovascular risk due to established CVD [2]. Therefore, despite their differences in threshold for diagnosis of hypertension, both guidelines are in agreement that treatment should be considered in patients with BPs in the range of 130 to $139 / 80$ to $89 \mathrm{mmHg}$ if they have high cardiovascular risk. Here, we discuss the evidence for drug treatment in this subset of patients, and the potential benefits and harms associated with antihypertensive treatment.

\section{REDEFINING THE THRESHOLD OF HYPERTENSION}

The differences in American and European guidelines regarding the BP threshold of hypertension stem from their differing definitions of hypertension. The American definition of hypertension is based on epidemiologic studies that convincingly show increased cardiovascular risk with increasing $\mathrm{BP}$; compared to those with a $\mathrm{BP}$ of $115 / 75 \mathrm{mmHg}$, each increase in SBP/DBP of $20 / 10 \mathrm{mmHg}$ is associated with double the risk of death from stroke or coronary artery disease (CAD) $[1,3]$. Additionally, meta-analyses of observational studies show a 1.5- and 2-fold higher risk of coronary heart disease and stroke, respectively, in subjects with BP 130 to $139 / 85$ to $89 \mathrm{mmHg}$ compared to those with $\mathrm{BP}<120 / 80 \mathrm{mmHg}$ [1,4-6]. A recent analysis of the Korea Acute Myocardial Infarction Registry revealed significant elevation of the odds ratio (OR) for 6-month major adverse cardiovascular events (MACE) in emergency department patients with high-normal BP and acute myocardial infarction (OR, 1.147; 95\% confidence interval [CI], 1.011 to 1.402; $p$ $=0.045)$ compared to patients with normal BP [7].

The American guideline defines hypertension as the BP threshold, above which the CVD risk is significantly increased compared to that associated with normal BP (130/80 mmHg) [1]. In contrast, the European guideline defines hypertension as the level of BP, above which the benefits of treatment (either with lifestyle interventions or drug treatment) clearly outweigh the risks of treatment [2]. Irrespective of the American or European definitions of hypertension, due to the high prevalence of prehypertension in the general population, lowering the threshold of hypertension would result in marked elevation in the prevalence of hypertension and an unnecessary increase in the risk of labeling a large portion of the population with a disease that they may not actually have $[8,9]$.

Prior to the Systolic Blood Pressure Intervention Trial (SPRINT) study, no trials used the SBP inclusion criteria of $\geq 130 \mathrm{mmHg}$ to investigate whether further lowering of BP would result in reduced cardiovascular risk [10]. However, the inclusion criteria and BP measurement methods used in the SPRINT trial suggest that the study did not actually include treatment-naïve patients with SBP > $130 \mathrm{mmHg}$ [10]. First, the majority of the patients enrolled in the SPRINT study were treated hypertensive patients, and only $2.7 \%$ of the patients in the intensive treatment arm and $11.3 \%$ in the standard treatment arm were considered treatment-naïve at randomization. As such, if these patients had been washed out from their medications, they would likely have had BP > 140/90 mmHg. Second, the SPRINT study used unattended automatic office blood pressure (AOBP) measurement for the office BP measurement. As studies have shown that AOBP is 5 to $15 \mathrm{mmHg}$ lower than manual office BP measurements, AOBP SBP of $130 \mathrm{mmHg}$ would have most likely corresponded to the manual office SBP of $140 \mathrm{mmHg}$, which would not differ from the previous definition of hypertension $[2,11]$. 
The criteria for hypertension differ depending on whether the American or European guideline definition of hypertension is used. Another important and controversial topic is whether these subjects should be treated with antihypertensive medications. The lines of evidence that support and dispute these recommendations are presented below.

\section{PROS AND CONS OF MEDICAL TREATMENT OF SUBJECTS WITH SBP BETWEEN 130-139 MMHG}

The benefit of BP lowering treatment in patients with hypertension is indisputable. In a recent meta-analysis of 123 studies including 613,815 participants, Ettehad et al. [12] demonstrated that each $10 \mathrm{mmHg}$ reduction in SBP is associated with a $20 \%$ reduction in the risk of major cardiovascular events, $17 \%$ reduction in the risk of coronary heart disease, $27 \%$ reduction in the risk of stroke, $28 \%$ reduction in the risk of heart failure, and $13 \%$ reduction in all-cause mortality. However, an unanswered question is whether BP lowering with anti-hypertensive treatment improves the outcome in patients with high-normal BP, defined as SBP between 130 to $139 \mathrm{mmHg}$. The most compelling evidence regarding the need to treat patients with SBP above 130 $\mathrm{mmHg}$ was provided by the SPRINT study, in which high-risk individuals with $\mathrm{BP}>130 \mathrm{mmHg}$ were randomized to a target SBP of 120 or $140 \mathrm{mmHg}$ [10]. The inclusion criteria of the SPRINT study were individuals $>50$ years of age with at least one of the following: (1) history of clinical/subclinical CVD, excluding stroke; (2) Framingham risk score $>15$; (3) aged $\geq 75$ years; or (4) CKD with estimated glomerular filtration rate between 20 to $60 \mathrm{~mL} / \mathrm{min} / 1.73 \mathrm{~m}^{2}$. However, as stated earlier, due to both the inclusion criteria and the method of office BP measurement, it is unclear whether the majority of the study population enrolled in the SPRINT study would have had SBP > $140 \mathrm{mmHg}$ if the baseline medications had been washed out and manual office BP measurement methods had been used for BP measurements [10]. However, one finding of the SPRINT study was that further intensive lowering of $\mathrm{BP}$ in high-risk hypertensive patients was associated with decreased mortality and cardiovascular events. In addition, the potential benefits of intensive BP lowering in high-risk patients were demonstrated by Xie et al. [13], in a meta-analysis of 19 clinical trials including 44,989 patients. In that meta-analysis, the average cardiovascular event rate was approximately $0.9 \%$ per year, corresponding to $9 \%$ to $10 \%$ per 10 years, and the average BP of the intensive BP lowering arm and the usual treatment arm was $133 / 76$ and $140 / 81 \mathrm{mmHg}$, respectively [13]. The results showed that intensive BP lowering was associated with a $14 \%$ reduction in cardiovascular events, $13 \%$ reduction in myocardial infarction, $22 \%$ reduction in stroke, $10 \%$ reduction in albuminuria, and 19\% reduction in retinopathy progression without significant reduction in heart failure, cardiovascular death, total mortality, or end-stage renal disease [13]. Based on this meta-analysis, the 2017 American guideline recommends drug treatment for primary prevention in highrisk stage 1 hypertensive patients with an arteriosclerotic cardiovascular disease (ASCVD) risk score $\geq 10 \%$, and in all stage 1 hypertension patients with previous CVD. A recent meta-analysis by Brunstrom and Carlberg [14] showed the association of antihypertensive treatment with a significant reduction in MACE, stroke, and heart failure in clinical trials that lowered BP in patients with a mean SBP of $138 \mathrm{mmHg}$ and coronary heart disease. In addition, the aforementioned meta-analysis by Ettehad et al. [12] revealed an association of BP lowering with reduced risk of major cardiovascular events, coronary heart disease, stroke, heart failure, and all-cause mortality in subjects with baseline SBP between 130 to $139 \mathrm{mmHg}$. In the same meta-analysis, BP lowering significantly reduced coronary heart disease in patients with baseline history of CVD whose baseline SBP was $139 \mathrm{mmHg}$, and significantly reduced the risk of heart failure in patients with baseline history of CVD and baseline SBP of $138 \mathrm{mmHg}$ [12]. Therefore, antihypertensive treatment is indicated for (1) secondary prevention of CVD in individuals with previous CVD, particularly coronary heart disease, and SBP between 130 to 139 $\mathrm{mmHg}$; and (2) primary prevention of CVD in high-risk individuals with SBP between 130 to $139 \mathrm{mmHg}$.

Several lines of evidence suggest that the above findings should not be generalized to all individuals with SBP in the range of 130 to $139 \mathrm{mmHg}$. In the Heart Outcomes Prevention Evaluation 3 (HOPE-3) trial, 12,705 subjects at intermediate cardiovascular risk were 
randomized using a two-by-two design to receive Candesartan + hydrochlorothiazide, rosuvastatin or placebo [15]. Although antihypertensive medications failed to significantly reduce the primary endpoints, participants in the upper tertile of baseline SBP (> 143.5 $\mathrm{mmHg}$ ) had significantly lower rates of first coprimary outcomes (defined as the composite of cardiovascular mortality, non-fatal myocardial infarction, and non-fatal stroke), and second coprimary outcomes (defined as the composite of coprimary outcomes, resuscitated cardiac arrest, heart failure, and revascularization). However, antihypertensive treatment showed no benefit in subjects in the middle and lower tertiles of baseline SBP [15]. The results from the HOPE-3 study suggest that BP lowering with antihypertensive treatment does not prevent CVD in subjects with intermediate-to-low-risk CVD and $\mathrm{SBP}<140 \mathrm{mmHg}$. In a meta-analysis of BP treatment in individuals with high-normal and normal BP at baseline, subjects at high- to very-high cardiovascular risk derived benefit from antihypertensive treatment, with a $60 \%$ reduction in the risk of stroke (hazard ratio [HR], 0.40; 95\% CI, 0.20 to o.81). However, subjects with low-to-moderate risk of CVD had no significant reduction in stroke, coronary heart disease, heart failure, cardiovascular death, or all-cause mortality [16]. In the aforementioned meta-analysis by Brunstrom and Carlberg [14], 74 clinical trials including the data of 306,273 subjects were analyzed regarding the efficacy of antihypertensive treatment according to the baseline SBP. The results showed that antihypertensive treatment in patients with baseline $\mathrm{SBP} \geq 160 \mathrm{mmHg}$ was associated with a significant reduction in the risk of cardiovascular mortality, major cardiovascular events, coronary heart disease, stroke, and heart failure. Similarly, antihypertensive treatment in subjects with baseline SBP in the range of 140 to $159 \mathrm{mmHg}$ was associated with a significant reduction in the risk of major cardiovascular events and coronary heart disease. However, antihypertensive treatment in subjects with baseline $\mathrm{SBP}<140 \mathrm{mmHg}$ showed no association with reduced risk of cardiovascular mortality, major cardiovascular events, coronary heart disease, or stroke [14].

Another important consideration in antihypertensive treatment is the risk of adverse events. A meta-analysis of 50 randomized clinical trials, which provided data on adverse events attributed to BP lowering treatment, showed that a $24 \%$ reduction in major cardiovascular risk factors was associated with an $89 \%$ increase in the risk of discontinuation, which corresponds to the prevention of 33 major cardiovascular events and 84 excess discontinuations/1,000 patients for 5 years [17]. As such, the benefit of BP lowering in high-normal subjects should be considered with caution, and careful titration should be performed to minimize adverse events. In addition, excessive lowering of BP, particularly in patients at high risk of CAD, may increase mortality and cardiovascular events. In a pooled analysis of the ONTARGET and TRANSCEND trials, which included 30,937 subjects at high cardiovascular risk, treating SBP $<120$ $\mathrm{mmHg}$ was associated with increased risk of composite cardiovascular outcome (HR, 1.14; 95\% CI, 1.03 to 1.26), cardiovascular death (HR, 1.29; 95\% CI, 1.12 to 1.49), and all-cause death (HR, 1.28; 95\% CI, 1.15 to 1.42) compared to treated subjects with SBP between 120 to $140 \mathrm{mmHg}$ [18]. Furthermore, a mean DBP $<70 \mathrm{mmHg}$ was associated with increased risks of composite primary outcome (HR, 1.31; 95\% CI, 1.20 to 1.42), myocardial infarction (HR, 1.55; $95 \% \mathrm{CI}, 1.33$ to 1.80), hospitalization for heart failure (HR, 1.59; 95\% CI, 1.36 to 1.86), and all-cause mortality (HR, 1.16; 95\% CI, 1.06 to 1.28) compared to subjects with a mean DBP in the range of 70 to $80 \mathrm{mmHg}$ [18]. Based on these data, the 2018 European Society of Cardiology/ European Society of Hypertension (ESC/ESH) guideline recommends that treated $\mathrm{BP}$ should not be lowered below $120 / 70 \mathrm{mmHg}[2]$. This recommendation is supported by a recent analysis of $1,234,435$ participants of the Korean Cancer Prevention Study cohort (789,255 males, aged 30 to 95 years) followed up for 22.5 million person-years. The study demonstrated that mortality from all-cause and ASCVD in those with DBP $<60 \mathrm{mmHg}$ compared to those with DBP between 70 to $79 \mathrm{mmHg}$ showed HRs of 1.23 (95\% CI, 1.16 to 1.30 ) and 1.37 (95\% CI, 1.20 to 1.57 ), respectively [19].

\section{CONCLUSIONS}

The current evidence suggests that antihypertensive treatment may be beneficial for subjects with SBP between 130 to $139 \mathrm{mmHg}$, for primary prevention in those at high risk of CVD and secondary prevention for those with coronary heart disease. However, there is a 
narrow therapeutic window between 120 to $130 / 70$ to 80 $\mathrm{mmHg}$, below which there may be an increased risk of mortality and cardiovascular events. Therefore, careful titration of antihypertensive medications to lower SBP to $130 \mathrm{mmHg}$ or below should be performed while ensuring that there are no adverse events. In addition, care should be taken to titrate the dosage to maintain the $\mathrm{BP}$ above $120 / 70 \mathrm{mmHg}$. No benefit of antihypertensive treatment for primary prevention has been shown in subjects with SBP below $140 \mathrm{mmHg}$ at low to intermediate risk of CVD.

\section{Conflict of interest}

No potential conflict of interest relevant to this article was reported.

\section{Acknowledgments}

This research was supported by the Fire Fighting Safety \& 119 Rescue Technology Research and Development Program funded by National Fire Agency ("MPSS- FireSafety-2015-80").

\section{REFERENCES}

1. Whelton PK, Carey RM, Aronow WS, et al. 2017 ACC/AHA/ AAPA/ABC/ACPM/AGS/APhA/ASH/ASPC/NMA/PCNA guideline for the prevention, detection, evaluation, and management of high blood pressure in adults: executive summary: a report of the American College of Cardiology/American Heart Association Task Force on Clinical Practice Guidelines. J Am Soc Hypertens 2018;12:579.e1579.e73.

2. Williams B, Mancia G, Spiering W, et al. 2018 ESC/ESH guidelines for the management of arterial hypertension. Eur Heart J 2018;39:3021-3104.

3. Lewington S, Clarke R, Qizilbash N, Peto R, Collins R; Prospective Studies Collaboration. Age-specific relevance of usual blood pressure to vascular mortality: a meta-analysis of individual data for one million adults in $6_{1}$ prospective studies. Lancet 2002;360:1903-1913.

4. Guo X, Zhang X, Guo L, et al. Association between pre-hypertension and cardiovascular outcomes: a systematic review and meta-analysis of prospective studies. Curr Hypertens Rep 2013;15:703-716.

5. Lee M, Saver JL, Chang B, Chang KH, Hao Q, Ovbiagele B.
Presence of baseline prehypertension and risk of incident stroke: a meta-analysis. Neurology 2011;77:1330-1337.

6. Wang $\mathrm{S}, \mathrm{Wu} \mathrm{H}$, Zhang $\mathrm{Q}, \mathrm{Xu}$ J, Fan Y. Impact of baseline prehypertension on cardiovascular events and all-cause mortality in the general population: a meta-analysis of prospective cohort studies. Int J Cardiol 2013;168:48574860.

7. Yoon NS, Jeong MH, Ahn Y, et al. Impact of high-normal blood pressure measured in emergency room on adverse cardiac events in acute myocardial infarction. Korean Circ J 2012;42:304-310.

8. Jang SY, Kim S, Lee CK, Cho EJ, Cho SJ, Lee SC. Prehypertension and left ventricular diastolic dysfunction in middle-aged Koreans. Korean Circ J 2016;46:536-541.

9. Khera R, Lu Y, Lu J, et al. Impact of 2017 ACC/AHA guidelines on prevalence of hypertension and eligibility for antihypertensive treatment in United States and China: nationally representative cross sectional study. BMJ 2018;362:k2357.

10. SPRINT Research Group, Wright JT Jr, Williamson JD, et al. A randomized trial of intensive versus standard blood-pressure control. N Engl J Med 2015;373:2103-2116.

11. Filipovsky J, Seidlerova J, Kratochvil Z, Karnosova P, Hronova M, Mayer O Jr. Automated compared to manual office blood pressure and to home blood pressure in hypertensive patients. Blood Press 2016;25:228-234.

12. Ettehad D, Emdin CA, Kiran A, et al. Blood pressure lowering for prevention of cardiovascular disease and death: a systematic review and meta-analysis. Lancet 2016;387:957967.

13. Xie X, Atkins E, Lv J, et al. Effects of intensive blood pressure lowering on cardiovascular and renal outcomes: updated systematic review and meta-analysis. Lancet 2016;387:435-443.

14. Brunstrom M, Carlberg B. Association of blood pressure lowering with mortality and cardiovascular disease across blood pressure levels: a systematic review and meta-analysis. JAMA Intern Med 2018;178:28-36.

15. Lonn EM, Bosch J, Lopez-Jaramillo P, et al. Blood-pressure lowering in intermediate-risk persons without cardiovascular disease. N Engl J Med 2016;374:2009-2020.

16. Thomopoulos C, Parati G, Zanchetti A. Effects of blood-pressure-lowering treatment on outcome incidence. 12. Effects in individuals with high-normal and normal blood pressure: overview and meta-analyses of randomized trials. J Hypertens 2017;35:2150-216o. 
Park S. Benefits of antihypertensive treatment in patients with high-normal blood pressure

17. Thomopoulos C, Parati G, Zanchetti A. Effects of blood pressure lowering treatment in hypertension: 8. Outcome reductions vs. discontinuations because of adverse drug events: meta-analyses of randomized trials. J Hypertens 2016;34:1451-1463.

18. Bohm M, Schumacher H, Teo KK, et al. Achieved blood pressure and cardiovascular outcomes in high-risk pa- tients: results from ONTARGET and TRANSCEND trials. Lancet 2017;389:2226-2237.

19. Kimm H, Mok Y, Lee SJ, Lee S, Back JH, Jee SH. The J-curve between diastolic blood pressure and risk of allcause and cardiovascular death. Korean Circ J 2018;48:3647. 